\title{
Tosse Crônica e Fonoaudiologia
}

Carta aos Editores

Letter to the editors

Rodrigo Dornelas ${ }^{1,2}$ (C)

Vanessa Veis Ribeiro', ${ }^{1,3}$

Mara Behlau ${ }^{1,4}$

\section{Chronic Cough and Speech Therapy}

Prezadas editoras-chefes

Ana Luiza Gomes Pinto Navas, Anna Alice Figueiredo de Almeida e Stela Maris Aguiar Lemos,

O fonoaudiólogo brasileiro, prestes a comemorar 40 anos de regulamentação da profissão, debruça-se em um novo seguimento de atuação: a tosse crônica, o que já é reconhecido em outros países, como Austrália ${ }^{(1,2)}$ e Estados Unidos ${ }^{(3)}$.

$\mathrm{Na}$ Fonoaudiologia, a laringe tradicionalmente era estudada por duas áreas com atuação que envolvem sua função, a voz, enquanto função fonatória, e a disfagia, enquanto função de proteção de vias aéreas inferiores. O novo desafio para o fonoaudiólogo é contribuir na avaliação e no tratamento dos pacientes com tosse crônica refratária, uma área que envolve uma expertise específica e que, como todas as outras áreas, requer estudos e preparo por parte do profissional. Nos casos de tosse crônica refratária, percebe-se que o foco principal é a laringe, o que permite uma sobreposição entre as especialidades voz e disfagia, com interesse direto na avaliação e tratamento desses pacientes. Indivíduos com tosse crônica podem ou não ter disfonia e/ou disfagia e, desta forma, os casos podem adquirir grande complexidade e exigir um raciocínio clínico mais estruturado, usando recursos combinados de uma abordagem híbrida que conta com elementos do modelo hipotético-dedutivo e da teoria da construção dos scripts das doenças, aplicados à intervenção fonoaudiológica ${ }^{(4)}$.

$\mathrm{Na}$ literatura, a primeira citação de tosse crônica ou conhecida na época também como tosse psicogênica foi realizada no início da década de $1980^{(5)}$. Logo em seguida, notase um interesse nessa área, com artigos publicados sobre as estratégias de avaliação e intervenção nos casos de pacientes com tosse crônica ${ }^{(1,6,7)}$. As evidências sobre os efeitos da intervenção fonoaudiológica começam a surgir e a reabilitação de pacientes com tosse, por fonoaudiólogos começa a ser reconhecida fora de nossa profissão, indicada por equipes multiprofissionais ${ }^{(1)}$.

A tosse é um mecanismo protetor resultante de um reflexo complexo, iniciado pela ativação de receptores irritantes na via aérea ${ }^{(8)}$. Trata-se de uma manobra expulsiva forçada, geralmente contra uma glote fechada ${ }^{(9)}$.

Quando sua duração é de até três semanas ela é considerada aguda e benéfica para o sistema respiratório por meio da remoção de substâncias nocivas e do aumento da depuração mucocilar, porém, a tosse crônica não tem benefício para o sistema respiratório ou para o corpo em geral ${ }^{(8)}$. Ao persistir por um período superior a oito semanas, a tosse passa a ser crônica. Uma exceção à regra ocorre nos casos de infecções do trato respiratório superior, em que a tosse por até oito semanas é considerada aceitável ${ }^{(10)}$. Para ser denominada de refratária, a tosse precisa ser persistente ao tratamento médico para causas específicas como doenças respiratórias e refluxo gastroesofágico ${ }^{(11)}$. A tosse crônica refratária é um problema difícil frequentemente associado ao aumento da

Trabalho realizado na Universidade Federal de São Paulo - UNIFESP - São Paulo (SP), Brasil.

${ }^{1}$ Universidade Federal de São Paulo - UNIFESP - São Paulo (SP), Brasil.

${ }^{2}$ Universidade Federal do Rio de Janeiro - UFRJ - Rio de Janeiro (RJ), Brasil.

${ }^{3}$ Universidade Federal de Sergipe - UFS - Lagarto (SE), Brasil.

${ }^{4}$ Centro de Estudos da Voz - CEV - São Paulo (SP), Brasil.

Fonte de financiamento: nada a declarar.

Conflito de interesses: nada a declarar. 
sensibilidade do reflexo da tosse ${ }^{(7)}$, e ocorre em até $46 \%$ dos pacientes com tosse crônica ${ }^{(2,12)}$. As principais características da tosse crônica refratária são sensação anormal de garganta ou cócegas (parestesia laríngea), aumento da sensibilidade à tosse em resposta a agentes tussígenos (hipertensão) e tosse desencadeada por estímulos não tussígenos, como falar ou ar frio (alotussia) ${ }^{(13)}$. Além disso, até $40 \%$ das pessoas com esse quadro sofrem problemas vocais e cerca de $56 \%$ também podem apresentar movimento paradoxal das pregas vocais ${ }^{(2)}$.

A avaliação fonoaudiológica dá-se por meio de procedimentos clínicos (exame clínico do paciente; frequência e limiar da tosse; avaliação perceptivo-auditiva, acústica e aerodinâmica da voz), além de instrumentos de autoavaliaçãoo ${ }^{(3,7,14,15)}$. Os questionários já validados em português brasileiro são o Índice de Severidade da Tosse (CSI-Br) que mensura a autopercepção da severidade dos sintomas de tosse $\mathrm{e}^{(16)}$, e o Questionário Newcastle de Hipersensibilidade Laríngea (LHQ-Br) que mensura a autopercepção de sensações laríngeas associadas com a síndrome da hipersensibilidade laríngea ${ }^{(17)}$. Já os protocolos traduzidos e adaptados para o Português Brasileiro são o Índice de Desvantagem Vocal - Garganta (IDV-G) ${ }^{(18)}$ que mensura a desvantagem vocal percebida relacionada aos sintomas de garganta e o Questionário de Leicester ${ }^{(19)}$ que avalia o sintoma de tosse e seu impacto no estado de saúde dos portadores de tosse crônica, sendo que ambos já encontram-se em processo de validação para o português brasileiro, com definição de suas propriedades psicométricas.

A reabilitação fonoaudiológica tem-se mostrado uma intervenção potencialmente eficiente no manejo da tosse crônica refratária e busca quebrar o ciclo de irritação dos receptores de tosse, quando a intervenção médica falha ${ }^{(8)}$, podendo ou não ser associada ao uso de fármacos anti-tussígenos ${ }^{(20-2)}$. As abordagens de tratamento para a tosse crônica refratária incluem supressão ativa da tosse, redução da sensibilidade ao reflexo da tosse ou aumento do limiar do reflexo da tosse ${ }^{(7,23)}$, além de redução da irritação laríngea ${ }^{(7)}$.

Recentemente foi elaborada uma proposta de reabilitação fonoaudiológica brasileira denominada Programa de Terapia para Manejo da Tosse Crônica (TMTC) para tratamento da tosse crônica refratária ${ }^{(24)}$. A literatura internacional apresenta outros programas como o physiotherapy, and speech and language therapy intervention (PSALTI) ${ }^{(25)}$ e o SPEech Pathology Intervention Program for CHronic Cough (SPEICH-C) ${ }^{(26)}$.

Observa-se assim que a Fonoaudiologia brasileira mostrase preocupada em obter validação de instrumentos e produzir evidências para explorar essa importante e promissora área de atuação, a fim de contribuir para a melhora da qualidade de vida de pacientes com tosse crônica refratária. Fonoaudiólogos especialistas em voz e em disfagia tem potencialmente a responsabilidade de contribuir no diagnóstico e tratamento dessa importante disfunção laríngea.

\section{REFERENCIAS}

1. Gibson PG, Vertigan AE. Speech pathology for chronic cough: a new approach. Pulm Pharmacol Ther. 2009;22(2):159-62. http://dx.doi. org/10.1016/j.pupt.2008.11.005. PMid:19061964.

2. Ryan NM, Gibson PG. Recent additions in the treatment of cough. J Thorac Dis. 2014;6(suppl. 7):S739-47. PMid:25383209.

3. Carroll TL. Chronic cough. San Diego, CA: LOGO Plural Publishing; 2019. $220 \mathrm{p}$

4. Peixoto JM, Santos SME, Faria RMD. Clinical reasoning development in medical students. Rev Bras Educ Med. 2018;42(1):73-81.

5. Blager FB, Gay ML, Wood RP. Voice therapy techniques adapted to treatment of habit cough: a pilot study. J Commun Disord. 1988;21(5):393-400. http:// dx.doi.org/10.1016/0021-9924(88)90024-X. PMid:3183084.

6. Vertigan AE, Theodoros DG, Gibson PG, Winkworth AL. Efficacy of speech pathology management for chronic cough: a randomised placebo controlled trial of treatment efficacy. Thorax. 2006;61(12):1065-9. http:// dx.doi.org/10.1136/thx.2006.064337. PMid:16844725.

7. Ryan NM, Vertigan AE, Bone S, Gibson PG. Cough reflex sensitivity improves with speech language pathology management of refractory chronic cough. Cough. 2010;6(1):5. http://dx.doi.org/10.1186/1745-99746-5. PMid:20663225.

8. Vertigan AE, Theodoros DG, Gibson PG, Winkworth AL. The relationship between chronic cough and paradoxical vocal fold movement: a review of the literature. J Voice. 2006;20(3):466-80. http://dx.doi.org/10.1016/j. jvoice.2005.08.001. PMid:16274959.

9. Morice AH, McGarvey L, Pavord I. Recommendations for the management of cough in adults. Thorax. 2006;61(suppl. 1):i1-24. http://dx.doi.org/10.1136/ thx.2006.065144. PMid:16936230.

10. Poulose V, Mohd IB. Prolonged cough presenting with diagnostic difficulty: a study of aetiological and clinical outcomes. Singapore Med J. 2011;52(4):267-70. PMid:21552788.

11. Chung KF, Pavord ID. Prevalence, pathogenesis, and causes of chronic cough. Lancet. 2008;371(9621):1364-74. http://dx.doi.org/10.1016/S01406736(08)60595-4. PMid:18424325.

12. Vertigan AE, Kapela SM, Franke I, Gibson PG. The effect of a vocal loading test on cough and phonation in patients with chronic cough. J Voice. 2017;31(6):76372. http://dx.doi.org/10.1016/j.jvoice.2017.03.020. PMid:28461166.

13. Ryan NM, Birring SS, Gibson PG. Gabapentin for refractory chronic cough: a randomised, double-blind, placebo-controlled trial. Lancet. 2012;380(9853):15839. http://dx.doi.org/10.1016/S0140-6736(12)60776-4. PMid:22951084.

14. Vertigan AE, Bone SL, Gibson PG. Development and validation of the Newcastle laryngeal hypersensitivity questionnaire. Cough. 2014;10(1):1. http://dx.doi.org/10.1186/1745-9974-10-1. PMid:24552215.

15. Vertigan A, Gibson P. Speech Pathology management of Cronic Refractory Cough and related disorders. Oxford, UK: Coptom Publishing; 2016.

16. Ribeiro VV, Lopes LW, Silva ACF, Medeiros AH No, Gartner-Schmidt J, Behlau M. Cough severity index: validation in Brazilian Portuguese. J Voice. [Internet]. 2021 Jul [citado em 2021 Maio 5]. Disponível em: https:// www.sciencedirect.com/science/article/abs/pii/S0892199721002022

17. Ribeiro VV, Lopes LW, Silva ACF, Medeiros AH No, Vertigan A, Behlau M. Validation of Newcastle Laryngeal Hypersensitivity Questionnaire (LHQ-Br) in Brazilian Portuguese. J Voice. [Internet]. 2021 Jul [citado em 2021 Maio 5]. Disponível em: https://www.sciencedirect.com/science/ article/abs/pii/S0892199721002009

18. Ribeiro VV, Lopes LW, Silva ACF, Medeiros AHNo, Lyberg-Åhlander V, Schalen L, et al. Voice handicap index-throat: translation and cross-cultural adaptation to Brazilian Portuguese. J Voice. [Internet]. 2020 Maio [citado em 2021 Maio 5] Disponível em: https://inkinghub.elsevier.com/retrieve/pii/S0892199720301338

19. Felisbino MB, Steidle LJM, Gonçalves-Tavares M, Pizzichini MMM, Pizzichini E. Leicester Cough Questionnaire: translation to Portuguese and cross-cultural adaptation for use in Brazil. J Bras Pneumol. 2014;40(3):21321. http://dx.doi.org/10.1590/S1806-37132014000300003. PMid:25029643.

20. Vertigan AE, Kapela SL, Ryan NM, Birring SS, McElduff P, Gibson PG. Pregabalin and speech pathology combination therapy for refractory chronic 
cough a randomized controlled trial. Chest. 2016;149(3):639-48. http:// dx.doi.org/10.1378/chest.15-1271. PMid:26447687.

21. Gibson P, Wang G, McGarvey L, Vertigan AE, Altman KW, Birring SS, et al. Treatment of unexplained chronic cough chest guideline and expert panel report. Chest. 2016;149(1):27-44. http://dx.doi.org/10.1378/chest.15-1496. PMid:26426314.

22. Chamberlain S, Birring SS, Garrod R. Nonpharmacological interventions for refractory chronic cough patients: systematic review. Lung. 2014;192(1):7585. http://dx.doi.org/10.1007/s00408-013-9508-y. PMid:24121952.

23. Chamberlain S, Garrod R, Birring SS. Cough suppression therapy: does it work? Pulm Pharmacol Ther. 2013;26(5):524-7. http://dx.doi.org/10.1016/j. pupt.2013.03.012. PMid:23524013.
24. Ribeiro VV, Lopes LW, Behlau M. Presentation of the Therapy Program for Management of Chronic Cough. CoDAS. 2021;33(3):e20200057. http:// dx.doi.org/10.1590/2317-1782/20202020057. PMid:34076101.

25. Mitchell SAFC, Garrod R, Clark L, Douiri A, Parker SM, Ellis J, et al. Physiotherapy, and speech and language therapy intervention for patients with refractory chronic cough: a multicentre randomised control trial. Thorax. 2017;72(2):129-36. http://dx.doi.org/10.1136/thoraxjnl-2016-208843. PMid:27682331.

26. Vertigan AE, Theodoros DG, Winkworth AL, Gibson PG. A comparison of two approaches to the treatment of chronic cough: perceptual, acoustic, and electroglottographic outcomes. J Voice. 2008;22(5):581-9. http://dx.doi org/10.1016/j.jvoice.2007.01.001. PMid:17485195. 


\title{
Errata: tosse crônica e fonoaudiologia
}

\section{Erratum: chronic coughand speech therapy}

\author{
Rodrigo Dornelas ${ }^{1,2}$, Vanessa Veis Ribeiro ${ }^{1,3}$, Mara Behlau ${ }^{1,4}$ \\ ${ }^{1}$ Universidade Federal de São Paulo - UNIFESP - São Paulo (SP), Brasil. \\ ${ }^{2}$ Universidade Federal do Rio de Janeiro - UFRJ - Rio de Janeiro (RJ), Brasil. \\ ${ }^{3}$ Universidade Federal de Sergipe - UFS - Lagarto (SE), Brasil. \\ ${ }^{4}$ Centro de Estudos da Voz - CEV - São Paulo (SP), Brasil.
}

Devido a problemas técnicos durante a produção editorial do artigo "Tosse Crônica e Fonoaudiologia", DOI https://doi.org/10.1590/2317-1782/20212021127, publicado em CoDAS, 34(1), e20210127, 2022, foi publicado com o erro de perda de espaços aleatórios em partes do texto.

No título em inglês das versões em português e inglês, onde se lê:

Chronic Coughand Speech Therapy

Leia-se:

Chronic Cough and Speech Therapy

No endereço para correspondência da versão em inglês, onde se lê:

RuaBotucatu, 802, Vila

Clementino, São Paulo (SP), Brasil,

CEP: 04023-062

Leia-se:

Rua Botucatu, 802, Vila

Clementino, São Paulo (SP), Brasil,

CEP: 04023-062

Na página 1 da versão em inglês:

O título e o tipo de artigo estão apresentados com o idioma em ordem invertida.

Nas referências das versões em português e inglês, onde se lê:

1. GibsonPG, VertiganAE. Speech pathology for chroniccough: a new approach. PulmPharmacolTher. 2009;22(2):159-62. http://dx.doi.org/10.1016/j.pupt.2008.11.005. PMid:19061964.

2. RyanNM, GibsonPG. Recentadditions in thetreatmentofcough.J ThoracDis. 2014;6(suppl. 7):S739-47. PMid:25383209.

3. CarrollTL. Chroniccough.San Diego, CA: LOGO Plural Publishing; 2019. 220 p.

4. PeixotoJM, SantosSME, FariaRMD. Clinicalreasoningdevelopment in medical students.RevBrasEduc Med. 2018;42(1):73-81.

5. BlagerFB, GayML, WoodRP. Voice therapytechniquesadaptedtotreatmentofhabitcough: a pilotstudy.J CommunDisord. 1988;21(5):393-400. http://dx.doi.org/10.1016/00219924(88)90024-X. PMid:3183084.

6. VertiganAE, TheodorosDG, GibsonPG, WinkworthAL. Efficacyof speech pathology management for chroniccough: a randomised placebo controlledtrialoftreatmentefficacy. Thorax. 2006;61(12):1065-9. http://dx.doi.org/10.1136/thx.2006.064337. PMid:16844725. 
7. RyanNM, VertiganAE, BoneS, GibsonPG. Coughreflexsensitivity improves with speech languagepathology management ofrefractorychroniccough.Cough. 2010;6(1):5. http://dx.doi.org/10.1186/1745-9974-6-5. PMid:20663225.

8. VertiganAE, TheodorosDG, GibsonPG, WinkworthAL. The relationshipbetweenchroniccoughandparadoxical vocal foldmovement: a review oftheliterature.J Voice. 2006;20(3):466-80. http://dx.doi.org/10.1016/j.jvoice.2005.08.001. PMid:16274959.

9. MoriceAH, McGarveyL, PavordI. Recommendations for the management ofcough in adults. Thorax. 2006;61(suppl. 1):i124. http://dx.doi.org/10.1136/thx.2006.065144. PMid:16936230.

10. PouloseV, MohdIB. Prolongedcoughpresentingwithdiagnosticdifficulty: a studyofaetiologicalandclinicaloutcomes.Singapore Med J. 2011;52(4):267-70. PMid:21552788.

11. ChungKF, PavordID. Prevalence, pathogenesis, and causes ofchroniccough.Lancet. 2008;371(9621):1364-74. http://dx.doi. org/10.1016/S0140-6736(08)60595-4. PMid:18424325.

12. VertiganAE, KapelaSM, FrankeI, GibsonPG. The effectof a vocal loadingtestoncoughandphonation in patientswithchroniccough.J Voice. 2017;31(6):763-72. http://dx.doi.org/10.1016/j.jvoice.2017.03.020. PMid:28461166.

13. RyanNM, BirringSS, GibsonPG. Gabapentin for refractorychroniccough: a randomised, double-blind, placebo-controlledtrial. Lancet. 2012;380(9853):1583-9. http://dx.doi.org/10.1016/S0140-6736(12)60776-4. PMid:22951084.

14. VertiganAE, BoneSL, GibsonPG. Developmentandvalidationofthe Newcastle laryngealhypersensitivityquestionnaire. Cough. 2014;10(1):1. http://dx.doi.org/10.1186/1745-9974-10-1. PMid:24552215.

15. VertiganA, GibsonP. Speech Pathology management ofCronicRefractoryCoughandrelateddisorders.Oxford, UK: CoptomPublishing; 2016.

16. RibeiroVV, LopesLW, SilvaACF, MedeirosAHNo, Gartner-SchmidtJ, BehlauM. Coughseverity index: validation in BrazilianPortuguese. J Voice. [Internet]. 2021 Jul [citado em 2021 Maio 5]. Disponível em: https://www.sciencedirect.com/science/ article/abs/pii/S0892199721002022

17. RibeiroVV, LopesLW, SilvaACF, MedeirosAHNo, VertiganA, BehlauM. Validationof Newcastle LaryngealHypersensitivityQuestionnaire (LHQ-Br) in BrazilianPortuguese. J Voice. [Internet]. 2021Jul [citado em 2021 Maio 5]. Disponível em: https://www.sciencedirect. com/science/article/abs/pii/S0892199721002009

18. RibeiroVV, LopesLW, SilvaACF, MedeirosAHNo, Lyberg-ÅhlanderV, SchalenL, et al. Voice handicap index-throat: translationandcross-cultural adaptationtoBrazilianPortuguese. J Voice. [Internet]. 2020Maio [citado em 2021 Maio 5]. Disponível em: https://linkinghub.elsevier.com/retrieve/pii/S0892199720301338

19. FelisbinoMB, SteidleLJM, Gonçalves-TavaresM, PizzichiniMMM, PizzichiniE. Leicester CoughQuestionnaire: translationtoPortugueseandcross-cultural adaptation for use in Brazil.J BrasPneumol. 2014;40(3):213-21. http://dx.doi.org/10.1590/ S1806-37132014000300003. PMid:25029643.

20. VertiganAE, KapelaSL, RyanNM, BirringSS, McElduffP, GibsonPG. Pregabalinand speech pathologycombinationtherapy for refractorychroniccough a randomizedcontrolledtrial.Chest. 2016;149(3):639-48. http://dx.doi.org/10.1378/chest.15-1271. PMid:26447687.

21. GibsonP, WangG, McGarveyL, VertiganAE, AltmanKW, BirringSS, et al. Treatmentofunexplainedchroniccoughchestguidelineand expert panel report.Chest. 2016;149(1):27-44. http://dx.doi.org/10.1378/chest.15-1496. PMid:26426314.

22. ChamberlainS, BirringSS, GarrodR. Nonpharmacologicalinterventions for refractorychroniccoughpatients: systematic review.Lung. 2014;192(1):75-85. http://dx.doi.org/10.1007/s00408-013-9508-y. PMid:24121952.

23. ChamberlainS, GarrodR, BirringSS. Coughsuppressiontherapy: does it work?PulmPharmacolTher. 2013;26(5):524-7. http://dx.doi.org/10.1016/j.pupt.2013.03.012. PMid:23524013.

24. RibeiroVV, LopesLW, BehlauM. PresentationoftheTherapyProgram for Management ofChronicCough.CoDAS. 2021;33(3):e20200057. http://dx.doi.org/10.1590/2317-1782/20202020057. PMid:34076101.

25. MitchellSAFC, GarrodR, ClarkL, DouiriA, ParkerSM, EllisJ, et al. Physiotherapy, and speech andlanguagetherapyintervention for patientswithrefractorychroniccough: a multicentrerandomisedcontroltrial.Thorax. 2017;72(2):129-36. http://dx.doi.org/10.1136/ thoraxjnl-2016-208843. PMid:27682331.

26. VertiganAE, TheodorosDG, WinkworthAL, GibsonPG. A comparisonoftwo approaches tothetreatmentofchroniccough: perceptual, acoustic, andelectroglottographicoutcomes.J Voice. 2008;22(5):581-9. http://dx.doi.org/10.1016/j.jvoice.2007.01.001. PMid:17485195.

\section{Leia-se:}

1. Gibson PG, Vertigan AE. Speech pathology for chronic cough: a new approach. Pulm Pharmacol Ther. 2009;22(2):159-62. http://dx.doi.org/10.1016/j.pupt.2008.11.005. PMid:19061964.

2. Ryan NM, Gibson PG. Recent additions in the treatment of cough. J Thorac Dis. 2014;6(suppl. 7):S739-47. PMid:25383209.

3. Carroll TL. Chronic cough. San Diego, CA: LOGO Plural Publishing; 2019. 220 p.

4. Peixoto JM, Santos SME, Faria RMD. Clinical reasoning development in medical students. Rev Bras Educ Med. 2018;42(1):73-81. 
5. Blager FB, Gay ML, Wood RP. Voice therapy techniques adapted to treatment of habit cough: a pilot study. J Commun Disord. 1988;21(5):393-400. http://dx.doi.org/10.1016/0021-9924(88)90024-X. PMid:3183084.

6. Vertigan AE, Theodoros DG, Gibson PG, Winkworth AL. Efficacy of speech pathology management for chronic cough: a randomised placebo controlled trial of treatment efficacy. Thorax. 2006;61(12):1065-9. http://dx.doi.org/10.1136/thx.2006.064337. PMid:16844725.

7. Ryan NM, Vertigan AE, Bone S, Gibson PG. Cough reflex sensitivity improves with speech language pathology management of refractory chronic cough. Cough. 2010;6(1):5. http://dx.doi.org/10.1186/1745-9974-6-5. PMid:20663225.

8. Vertigan AE, Theodoros DG, Gibson PG, Winkworth AL. The relationship between chronic cough and paradoxical vocal fold movement: a review of the literature. J Voice. 2006;20(3):466-80. http://dx.doi.org/10.1016/j.jvoice.2005.08.001. PMid:16274959.

9. Morice AH, McGarvey L, Pavord I. Recommendations for the management of cough in adults. Thorax. 2006;61(suppl. 1):i1-24. http://dx.doi.org/10.1136/thx.2006.065144. PMid:16936230.

10. Poulose V, Mohd IB. Prolonged cough presenting with diagnostic difficulty: a study of aetiological and clinical outcomes. Singapore Med J. 2011;52(4):267-70. PMid:21552788.

11. Chung KF, Pavord ID. Prevalence, pathogenesis, and causes of chronic cough. Lancet. 2008;371(9621):1364-74. http:// dx.doi.org/10.1016/S0140-6736(08)60595-4. PMid:18424325.

12. Vertigan AE, Kapela SM, Franke I, Gibson PG. The effect of a vocal loading test on cough and phonation in patients with chronic cough. J Voice. 2017;31(6):763-72. http://dx.doi.org/10.1016/j.jvoice.2017.03.020. PMid:28461166.

13. Ryan NM, Birring SS, Gibson PG. Gabapentin for refractory chronic cough: a randomised, double-blind, placebo-controlled trial. Lancet. 2012;380(9853):1583-9. http://dx.doi.org/10.1016/S0140-6736(12)60776-4. PMid:22951084.

14. Vertigan AE, Bone SL, Gibson PG. Development and validation of the Newcastle laryngeal hypersensitivity questionnaire. Cough. 2014;10(1):1. http://dx.doi.org/10.1186/1745-9974-10-1. PMid:24552215.

15. Vertigan A, Gibson P. Speech Pathology management of Cronic Refractory Cough and related disorders. Oxford, UK: Coptom Publishing; 2016.

16. Ribeiro VV, Lopes LW, Silva ACF, Medeiros AH No, Gartner-Schmidt J, Behlau M. Cough severity index: validation in Brazilian Portuguese. J Voice. [Internet]. 2021 Jul [citado em 2021 Maio 5]. Disponível em: https://www.sciencedirect.com/ science/article/abs/pii/S0892199721002022

17. Ribeiro VV, Lopes LW, Silva ACF, Medeiros AH No, Vertigan A, Behlau M. Validation of Newcastle Laryngeal Hypersensitivity Questionnaire (LHQ-Br) in Brazilian Portuguese. J Voice. [Internet]. 2021 Jul [citado em 2021 Maio 5]. Disponível em: https:// www.sciencedirect.com/science/article/abs/pii/S0892199721002009

18. Ribeiro VV, Lopes LW, Silva ACF, Medeiros AH No, Lyberg-Åhlander V, Schalen L, et al. Voice handicap index-throat: translation and cross-cultural adaptation to Brazilian Portuguese. J Voice. [Internet]. 2020 Maio [citado em 2021 Maio 5]. Disponível em: https://inkinghub.elsevier.com/retrieve/pii/S0892199720301338

19. Felisbino MB, Steidle LJM, Gonçalves-Tavares M, Pizzichini MMM, Pizzichini E. Leicester Cough Questionnaire: translation to Portuguese and cross-cultural adaptation for use in Brazil. J Bras Pneumol. 2014;40(3):213-21. http://dx.doi. org/10.1590/S1806-37132014000300003. PMid:25029643.

20. Vertigan AE, Kapela SL, Ryan NM, Birring SS, McElduff P, Gibson PG. Pregabalin and speech pathology combination therapy for refractory chronic cough a randomized controlled trial. Chest. 2016;149(3):639-48. http://dx.doi.org/10.1378/ chest.15-1271. PMid:26447687.

21. Gibson P, Wang G, McGarvey L, Vertigan AE, Altman KW, Birring SS, et al. Treatment of unexplained chronic cough chest guideline and expert panel report. Chest. 2016;149(1):27-44. http://dx.doi.org/10.1378/chest.15-1496. PMid:26426314.

22. Chamberlain S, Birring SS, Garrod R. Nonpharmacological interventions for refractory chronic cough patients: systematic review. Lung. 2014;192(1):75-85. http://dx.doi.org/10.1007/s00408-013-9508-y. PMid:24121952.

23. Chamberlain S, Garrod R, Birring SS. Cough suppression therapy: does it work? Pulm Pharmacol Ther. 2013;26(5):524-7. http://dx.doi.org/10.1016/j.pupt.2013.03.012. PMid:23524013.

24. Ribeiro VV, Lopes LW, Behlau M. Presentation of the Therapy Program for Management of Chronic Cough. CoDAS. 2021;33(3):e20200057. http://dx.doi.org/10.1590/2317-1782/20202020057. PMid:34076101.

25. Mitchell SAFC, Garrod R, Clark L, Douiri A, Parker SM, Ellis J, et al. Physiotherapy, and speech and language therapy intervention for patients with refractory chronic cough: a multicentre randomised control trial. Thorax. 2017;72(2):129-36. http:// dx.doi.org/10.1136/thoraxjnl-2016-208843. PMid:27682331.

26. Vertigan AE, Theodoros DG, Winkworth AL, Gibson PG. A comparison of two approaches to the treatment of chronic cough: perceptual, acoustic, and electroglottographic outcomes. J Voice. 2008;22(5):581-9. http://dx.doi.org/10.1016/j.jvoice.2007.01.001. PMid:17485195.

Pedimos desculpas pelos erros. 